\title{
An Agent-Based Model of monocyte differentiation into Tumour-Associated Macrophages in Chronic Lymphocytic Leukemia
}

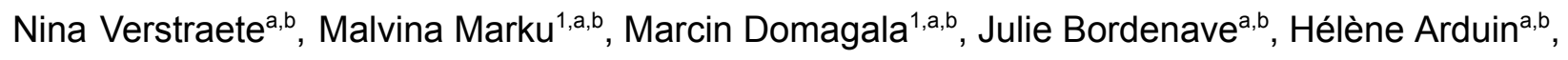
Jean-Jacques Fourniéa,b ${ }^{a}$ Loïc Ysebaert ${ }^{d}$, Mary Poupot ${ }^{a, b}$ and Vera Pancaldia,b,c

a INSERM, Cancer Research Center of Toulouse, 2 Avenue Hubert Curien, 31037, CEDEX 1 Toulouse, France.

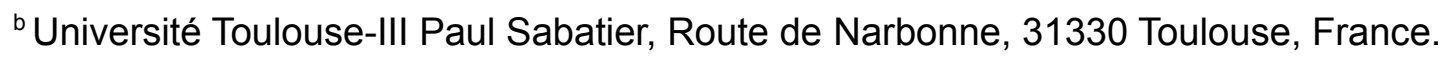

'Barcelona Supercomputing Center, Carrer de Jordi Girona, 29, 31, 08034 Barcelona, Spain.

d Service d'Hématologie, Institut Universitaire du Cancer de Toulouse-Oncopole, 31330 Toulouse, France.

${ }^{1}$ These authors contributed equally to this work.

\section{Keywords}

agent-based model, tumour-associated macrophage, chronic lymphocytic leukemia

\section{Abstract}

Monocyte-derived macrophages are immune cells which help maintain tissue homeostasis and defend the organism against pathogens. In solid tumours, recent studies have uncovered complex macrophage populations, among which tumour-associated macrophages, supporting tumorigenesis through multiple cancer hallmarks such as immunosuppression, angiogenesis or matrix remodelling. In the case of chronic lymphocytic leukemia, these macrophages are known as nurse-like cells and have been shown to protect leukemic cells from spontaneous apoptosis and contribute to their chemoresistance. We propose an agent-based model of monocytes differentiation into nurse-like cells upon contact with leukemic B cells in-vitro. We studied monocyte differentiation and cancer cells survival dynamics depending on diverse hypotheses on monocytes and cancer cells relative proportions, sensitivity to their surrounding environment and cell-cell interactions. Peripheral blood mononuclear cells from patients were cultured and monitored during 13 days to calibrate the model parameters, such as phagocytosis efficiency, death rates or protective effect from the nurse-like cells. Our model is able to reproduce experimental results and predict cancer cells survival dynamics in a patient-specific manner. Our 
results shed light on important factors at play in cancer cells survival, highlighting a potentially important role of phagocytosis.

\section{Introduction}

Advances in cancer therapies involving a better understanding of the role of immune cells inside tumours have led to a dramatic improvement of the response and survival of patients. These therapies are based on the reactivation of immune cells that normally detect and eliminate cancer cells and whose cytotoxic activity is inhibited within tumours. However, the great progress offered by these approaches is hampered by the ineffectiveness of these therapies in two thirds of cancer patients and by the resistance and associated relapse for some patients initially responding. Additionally, these immunotherapies can present a series of strong side effects ranging from rare deadly conditions to very uncomfortable autoimmune chronic conditions affecting some of the patients considered 'cured' from their cancers. The revolution of immune therapies resulted in a paradigm shift in our understanding of cancer and the more we discover about the various cells present in tumours and the specific ways in which they interact with each other, the better we will be able to tune the tumour microenvironment (TME) to arrest tumour growth.

In recent years, the research focus has been mostly on anti-tumoral T lymphocytes. In this project, we aim to better characterise the myeloid cells which protect the cancer cells from this attack by $T$ cells and promote tumour growth. It has long been known that tumours involve high levels of inflammation and for this reason macrophages are found in abundance in tumour biopsies. Macrophages can be either derived from monocytes in blood or tumour resident and are usually described as a pro-inflammatory (M1) or anti-inflammatory (M2) polarization state, depending on their en-vironmental signals (1) (2). However, recent single cell studies identified a broader spectrum of phenotypes also impacting their specific functions such as phagocytosis, immunoregulation, matrix deposition, tissue remodelling and tumour resistance. In tumours, macrophages can be educated by the cancer cells to promote their growth, becoming Tumour Associated Macrophages (TAMs) (3) (4). Activation of the TAM polarization pathway leads to the secretion of several cytokines, such as CXCL12/13, IL-10, and IL-6/IL-8, which are reported to have pro-tumoral effect, providing protection to the cancer cells.

A similar ecology of cancer cells and macrophages is established in the case of Chronic Lymphocytic Leukemia (CLL), a blood borne malignancy characterized by the accumulation of large quantities of CD19+/CD5+ B cancer cells. These cells can be encountered in the bloodstream but also in lymphoid organs (bone-marrow, spleen and lymph nodes) forming proliferating centers in which high densities of these cells are observed. B-CLL are unable to 
proliferate on their own and need to migrate to proliferation centers where they can encounter a supportive TME comprising T cells, stromal cells and TAMs which are called Nurse-like cells (NLCs) in this pathology. It has been widely reported that NLCs are crucial in rescuing B-CLL from spontaneous apoptosis and are important in attracting them to the proliferation centers.

Similarly to cancer cells in solid tumours, B-CLL cells are able to induce the differentiation of monocytes into NLCs through direct contact and cytokine production, which will favor the establishment of a pro-tumoral environment, protecting the leukemic cells from spontaneous apoptosis, often leading to therapy resistance(5). One of the limitations in the study of TAMs is the difficulty in identifying them in bulk tumour samples, due to their close similarity with other macrophages that are also present in the TME. However, we showed that although NLCs and M2-type macrophages display a similar profile in the CLL microenvironment (6), some distinctions can be highlighted, such as high expression of the RAGE membrane receptor, the HIF1 $a$ and VEGF/EGF transcription factors in NLCs (7). Given the nurturing properties of NLCs in the CLL microenvironment, a high number of NLCs has been reported to lead to disease progression and shorter overall survival (8),(9). It has also been reported that NLCs express high levels of stromal-derived factor 1-a (SDF-1a), a potent chemoattractant for CLL cells inducing their migration and emperipolesis, the presence of entire CLL cells inside macrophages (10). Beside release of soluble factors, NLCs can also rescue B-CLL by direct contact (11). Other molecules released by NLC such as BAFF, BDNF and APRIL have been reported to support survival of B-CLL (12),(13). However, the detailed mechanisms that lead to the formation of NLCs remain to be fully elucidated. Importantly, the formation of NLCs can be observed and studied in a biologically relevant in-vitro system in which patient-derived Peripheral Blood Mononuclear Cells (PBMC) are cultured for a few days. PBMCs are usually composed of $1-3 \%$ monocytes and $95 \%$ B-CLL cells, or more, depending on the patient and disease stage. Heterologous in -vitro co-cultures of healthy monocytes and patient-derived B-CLL cells can also be used to produce NLCs in the absence of any other cell type. These two systems constitute a great resource to dissect the detailed processes that take place during the differentiation of monocytes and their polarization into NLCs. Moreover, controllable conditions in this experiment allow a detailed investigation of the conditions that are necessary and sufficient for NLC production and make it a perfect tool to develop computational models of this process.

Agent-based models (ABMs) represent a discrete modelling approach that enables the simulation of the dynamics of populations of individuals in an environment. In principle, ABMs consist of decentralized agents, each of which is defined by its own characteristics and behaviour rules in space and time, whereas the global behaviour of the system emerges as a result of a community of diverse individual agents situated in a specific common environment 
(14). Importantly, contrary to other equation-based models (15) (16) (17), the dynamics (either deterministic or stochastic) is spatio-temporal, enabling the identification of spatial patterns of individuals in time, while modular agent classes can be defined and complex emergent properties can be studied (18). In cancer biology, ABMs have been widely used to simulate the dynamics of diverse immune and cancer cell populations (19) (20), as well as to simulate adaptation of cancer cells in the TME, mutations and phenotype diversity, cell plasticity, the role of the extracellular matrix, and the effect of drugs and on tumour survival and proliferation (21). Over the years, a number of computational tools forf implementing ABMs have been developed, including advanced methods for incorporating both inter- and intra-cellular interactions to simulate the global dynamics of multicellular systems (22) (23). Here, we present an ABM implemented in Netlogo (24) (25) aiming to reproduce monocyte differentiation and polarization into NLC upon contact with B-CLL cells in an in-vitro environment. We calibrate it on in-vitro cultures of CLL patients and perform extensive parameter optimization using parameter exploration based on genetic algorithms integrated in the OpenMOLE framework (26). These models allow us to gain quantitative insights into important factors and processes in this biological system, such as phagocytosis and anti-apoptotic signalling mechanisms that protect CLL cells from apoptosis.

\section{Results}

\section{NLC formation in-vitro}

In order to observe formation of NLCs in-vitro, autologous cultures from CLL patients PBMC were monitored during 13 days (Fig.1A). Daily observation allowed us to see outgrowth of big, adherent NLCs, whose phenotype was further assessed by flow cytometry. Additionally, concentration and viability of B-CLL were measured at the indicated time points (Fig.1C). Based on the changes in morphology and expression of myeloid markers of maturating NLC (CD11c, CD14, CD16, CD163 and CD206) and on the condition of B-CLL cells, we have distinguished 4 stages of the culture which allowed us to specify global and local behaviours in the ABM design. 
A

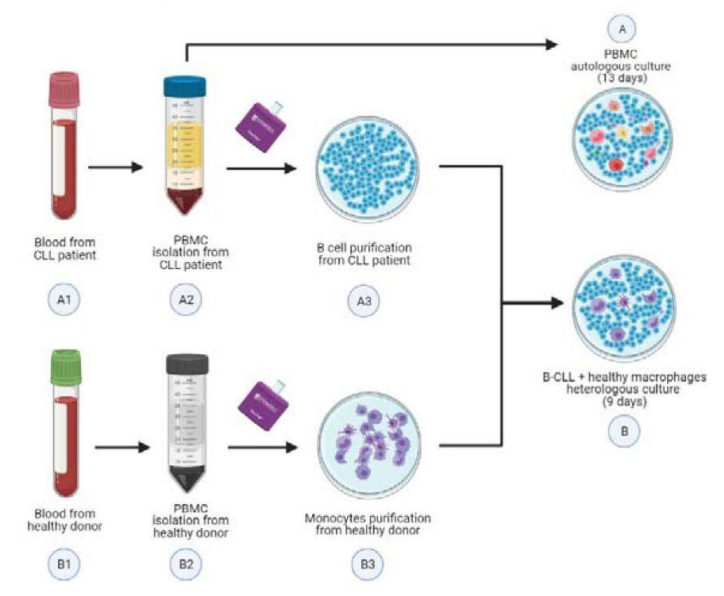

C B-CLL viability and concentration from PBMC in-vitro cultures
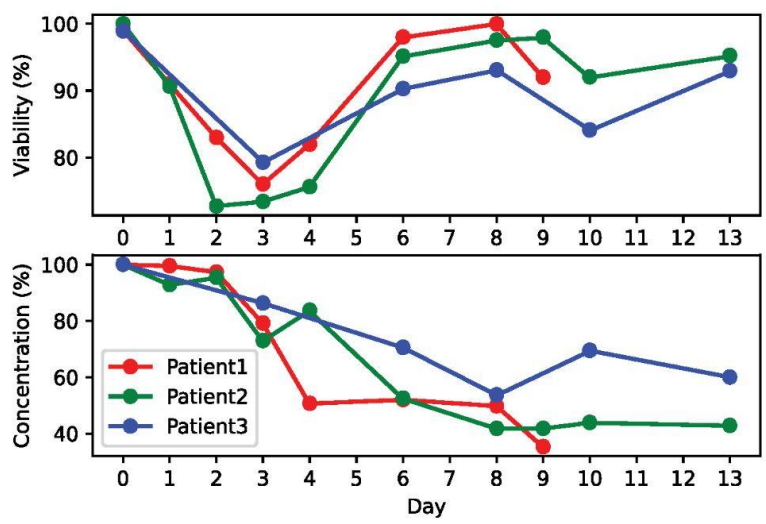

B
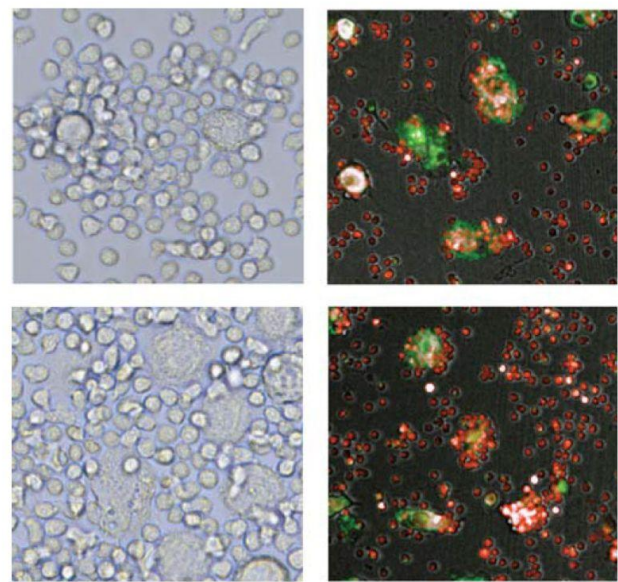

D B-CLL viability and concentration

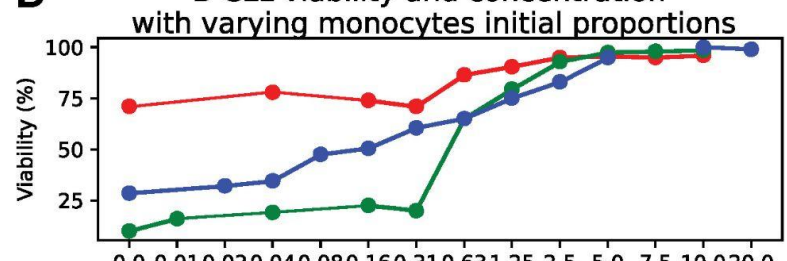

$0.0 \quad 0.010 .020 .040 .080 .160 .310 .631 .252 .5 \quad 5.0 \quad 7.5 \quad 10.020 .0$

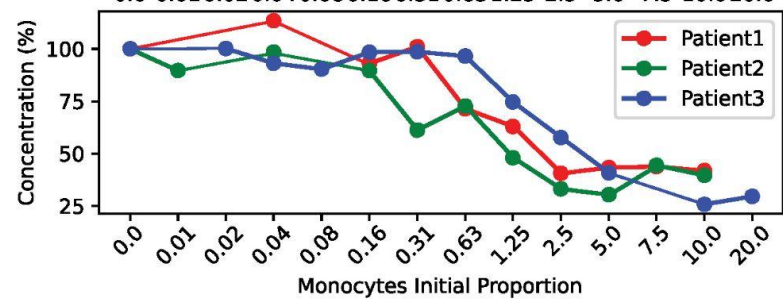

Fig. 1 Experimental setups and datasets from in-vitro CLL patient PBMC cultures. A) Experimental set-up for the experiments: Autologous ( $A$ ) and heterologous (B) co-cultures of B-CLL and monocytes leading to NLC production. In autologous cultures, Peripheral Blood Mononuclear Cells (PBMC) are isolated from CLL patients' blood samples and cultured invitro for 13 days. The cell concentration and viability of B-CLL was monitored at indicated time points by haemocytometer and flow cytometry AnnexinV/7AAD staining respectively. B) NLC formation as visualized in bright-field and immuno-fluorescence microscopy (. C) Time course datasets produced from the PBMC autologous cultures. B-CLL cells survival was monitored by viability assay and concentration measurements. D) Time course datasets produced from the heterologous co-cultures. Monocytes from healthy donors and B-CLL were co-cultured to assess the relationship between the initial density of monocytes and the level or survival of B-CLL after 9 days. The $x$-axis displays different monocytes initial proportions (not to scale for clarity). Measurements resulting from duplicates on the same patient were averaged.

- Day0-Day3: initial state of the culture characterized by increased apoptosis of B-CLL as a result of post-isolation stress and initial lack of pro-survival agents; monocytes attach to the plastic and start differentiation into macrophages (M0); based on the B-CLL cell number, the phagocytosis activity of differentiating monocytes is low (M1 or M2 is not depicted in detail, but a killing efficiency activity from the Macrophage class is explored $(0 \%$ to $5 \%$ probability that a macrophage phagocytes a living B-CLL). 
- Day3-Day6: continuation of maturation of macrophages/NLC; intensification of phagocytosis of the dead/apoptotic B-CLL cells (and of some living ones up to a certain extent); the global B-CLL viability rises and concentration starts to stabilize.

- Day6-Day10: macrophage/NLC reach maturation; we observe a tendency for B-CLL cells to accumulate around NLCs; B-CLL viability and cell concentration stabilizes.

- Day10-Day13: culture reaches the stabilization phase; B-CLL viability remains around $95 \%$ and cell concentration remains constant reaching $40-50 \%$ of the initial concentration.

\section{An agent-based model of NLC formation}

In this section, we describe the construction of a semi-quantitative ABM recapitulating the cell behaviours described in the previous section. The key concept is that the B-CLL survival dynamics observed in-vitro can be described as the evolution of a system composed by two main cell populations (Fig.2A) : cancer cells (B-CLL) and myeloid cells (monocytes, macrophages, NLCs). Cancer cells can be found in 3 possible states, depending on their life-counter : (i) need signal when they are still above the apoptosis threshold and are attracted by chemotaxis to anti-apoptotic signals from NLCs, (ii) apoptotic which is an irreversible state in which the cells will continue moving and eventually die and (iii) dead when the cells have reached the death threshold and will remain immobile. Myeloid cells can be found under 3 states as well, corresponding to their differentiation level : (i) monocyte, (ii) macrophage or (iii) NLC. Depending on their state, cells are characterized by specific properties and spatiotemporal behaviors in terms of different actions including movement, phagocytosis or cell-cell interactions (Fig. 2B). From the dynamics shown in Fig.1C, microscopy observations and flow cytometry data on the evolution of the NLC phenotype (data not shown), we deduced global behaviours that might be at play in the cells' interactions (see. Methods section for a detailed description of the rules). The model also has a series of parameters that could either be calibrated based on experimental observations or optimized through a parameter exploration that minimizes the difference between the predicted B-CLL cell viability and concentration and the corresponding experimental data from autologous PBMC culture throughout 13 days. The parameter optimization was performed on simulations of 1000 cells, including $1.6 \%$ monocytes (experimental estimation from 3 patients), and a total cell density of $55 \%$. 
A

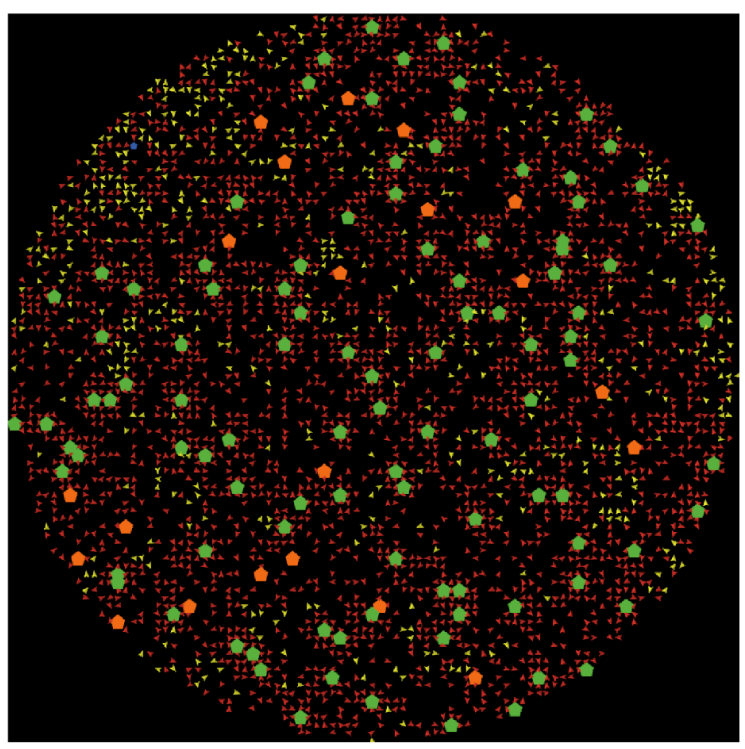

B

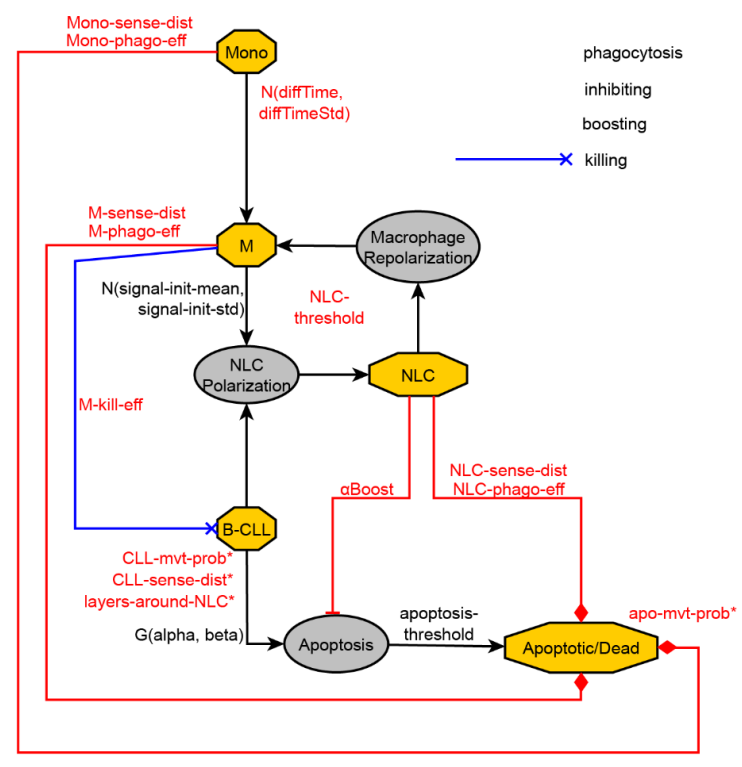

Fig. 2. An ABM model of monocyte differentiation into NLCs in an in-vitro culture of CLL cells. A). Netlogo simulation of 5000 cells in a 2D world. Cancer cells are depicted as small arrows (red, yellow or grey for need-signal, apoptotic or dead state, respectively) and myeloid cells are depicted as pentagons (small blue, large orange or green for monocytes, macrophages or NLCs, respectively). B) Schematic Diagram of the agents' behaviours. Parameters calibrated based on experimental data are written in black and parameters optimized through the genetic algorithm search are written in red (see Supplementary material for more details about the model elements).

\section{Parameter space exploration}

Based on the 2D ABM model of monocyte to NLC differentiation in presence of cancer B-CLL cells described in the previous section, we performed multiple simulations using various model parameters fixed to biologically reasonable values, allowing us to estimate the ranges to be systematically explored for each parameter (Supplementary Table). To determine the most effective parameter values to reproduce the B-CLL survival dynamics observed experimentally in the PBMC autologous culture, we then performed an optimization analysis employing the nondominated sorting genetic algorithm-II (NSGA-II, (27), (28), Fig. 3A) as implemented in openMOLE (26). This procedure systematically generates populations of parameter sets and evolves the candidate solutions toward higher values of two objective fitness functions corresponding to the optimization of the fit between experimental data and model predictions on both B-CLL viability and concentration dynamics. We next calculate the Pareto front to identify the sets of parameters which represent the best trade-offs between the two constraints (Fig. 3B).

A total of 45 parameter sets were obtained as best fitting solutions from the Pareto front. We find that our two objectives are conflicting, as shown by the convex shape of the pareto front solutions, with an increased fitness on viability leading to lower fitness on concentration and the 
reverse (Fig. 3B). In addition, we found that optimal parameters from the Pareto front are distributed within specific value ranges (Fig. 3C). Monocyte phagocytosis effciency is skewed towards low values, and macrophage phagocytosis efficiency towards high values, consistent with the biological observation that phagocytosis from non-adherent monocytes in the first days is negligible. We further analyzed correlations between parameters (Fig. 3D) and parameter correlation with our two fitness functions (Fig. 3E). This also suggests a strong role for phagocytosis (especially mono-phago-eff) in obtaining a higher viability fitness (Fig. 3E).

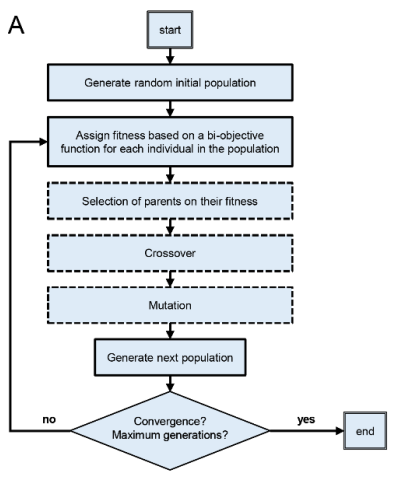

B

B

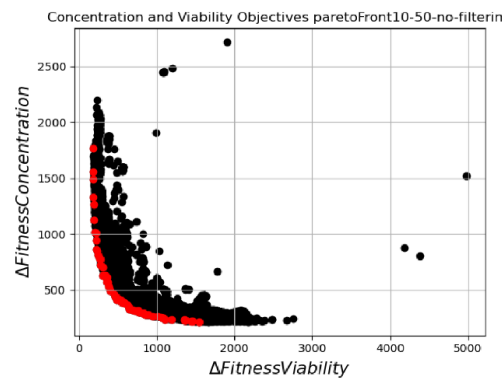

C

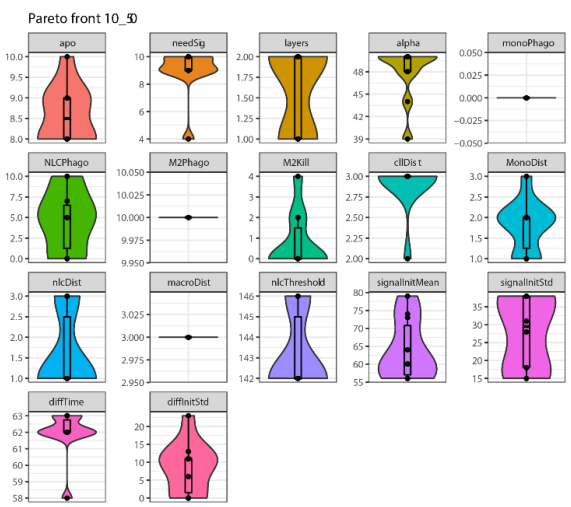

$\mathrm{D}$

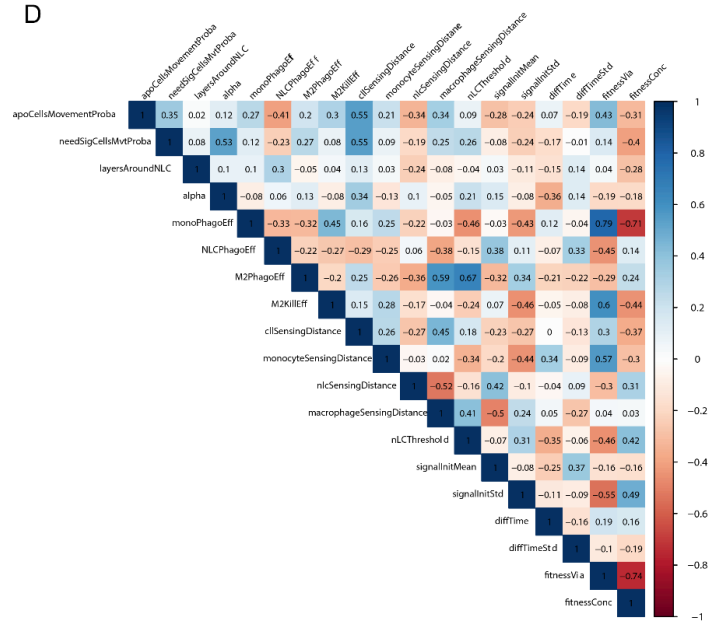

$E$

Fig. 3. Overview of strategy and results of the parameter exploration A) Schematic of the bi-objective genetic algorithm. B) Pareto front of the bi-objective search. The NSGA2 genetic algorithm evaluates each explored set of parameters (20'000 generations) according to 2 objective functions corresponding to B-CLL viability and concentration, throughout each simulation (10 time points, least squares method). The Pareto front depicted in red here contains a set of non-dominated solutions. C) Pareto front parameters distribution. D) Pairwise parameter correlation. Pearson's correlation coefficients were calculated pairwise based on the selected parameter values throughout the NSGA2 genetic algorithm for 20 '000 generations from the OpenMOLE output. E) Parameters correlations with fitnesses on cell viability (left panel) and cell concentration (right panel). Parameters are ranked based on the absolute value of their correlation coefficient with viability fitness. 


\section{Model validation and parameter sensitivity analysis}

Using the parameters set with the highest fitness on viability obtained from the exploration procedure, we observed a good fit of the simulation results compared to experimental data from B-CLL survival in PBMC autologous cultures from 3 different patients (Fig. 4A). The opposing constraints between fitness on viability and concentration might prevent the algorithm from converging towards best fitting solutions on each objective independently, which could explain the few discrepancies between model prediction and experimental data. The best solution was achieved with the set of parameter values given in Supplementary Table 1.

To test the predictive power of our model, we additionally ran simulations in which we changed the initial monocyte concentration in the heterologous cultures (see Fig. 1). We then performed experiments observing B-CLL survival in heterologous co-cultures with the corresponding varying monocyte proportions at a specific time point during the culture (Fig. 4B) and were able to verify the predicted viability and concentration values observed in the simulation. Finally, satisfactory predictions of B-CLL survival were obtained also when simulating cultures with patient-specific monocyte proportions (Fig. 4C). Finally, to better understand the influence of some specific parameters on the overall B-CLL survival dynamics, we performed simulations varying one parameter at a time from the best solution identified in the previous section (Fig. 4D). We first explored the importance of phagocytosis running simulations without any phagocytosis, and with decreasing monocyte, macrophage and NLC phagocytosis efficiency respectively, highlighting an important impact of these parameters on the B-CLL viability dynamics. Indeed, eliminating phagocytosis completely abolishes our ability to reproduce the experimentally observed dynamics. We further explored the importance of NLC protective effect on cancer cells running simulations with decreasing values of the corresponding protective chemical (alpha-boost) showing that its impact is observable mainly after 6 days of culture. Next, we evaluated the importance of the threshold that determines at which point a macrophage can be polarised into NLCand observed that it also strongly impacts the viability dynamics. The differentiation time of monocytes to macrophages was also shown to influence the survival dynamics as displayed by the simulations with varying mean and standard deviation values for the differentiation time at initialization. Other sources of variability in the initial distributions, such as initial signal strength in monocytes, seem to influence the dynamics

importantly. On the contrary, B-CLL movement probabilities and macrophage killing efficiency was shown to not have any considerable impact on B-CLL survival dynamics with this set of parameters. 
bioRxiv preprint doi: https://doi.org/10.1101/2021.12.17.473137; this version posted December 20, 2021. The copyright holder for this preprint (which was not certified by peer review) is the author/funder, who has granted bioRxiv a license to display the preprint in perpetuity. It is made available under aCC-BY 4.0 International license.

A
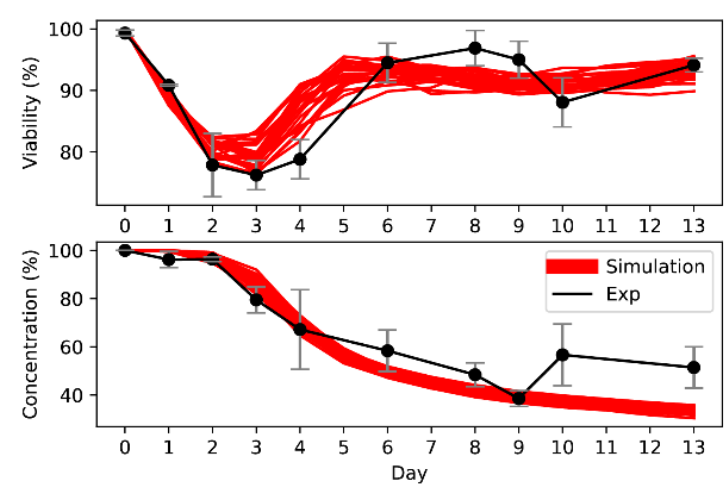

B

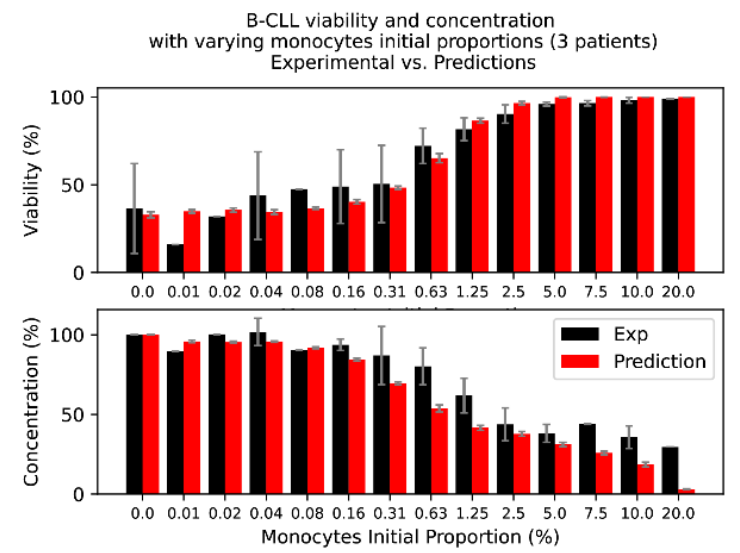

C
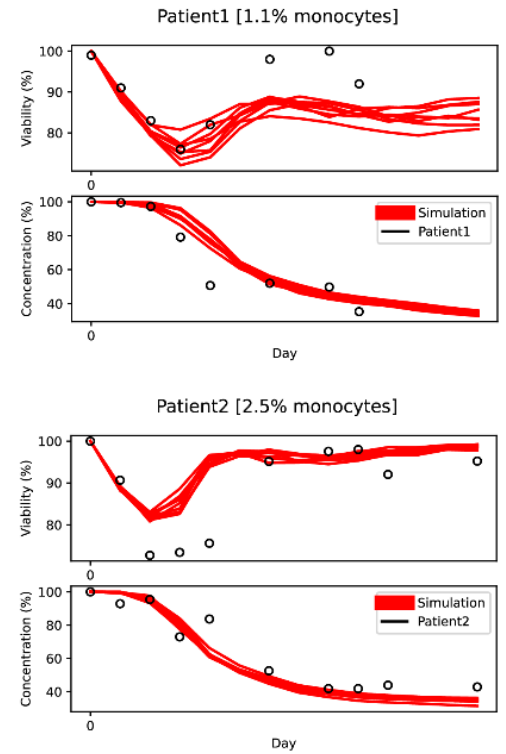

Day

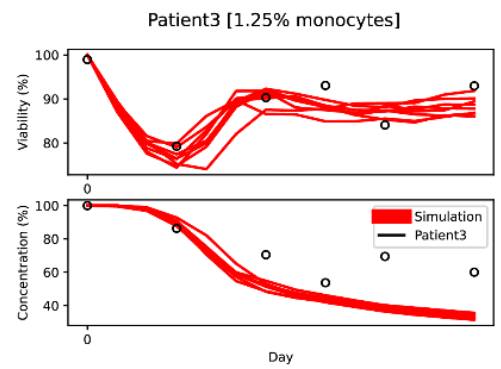

D
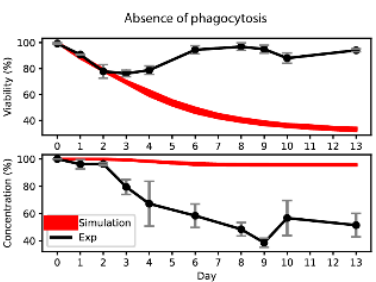

Initial Signal Streng
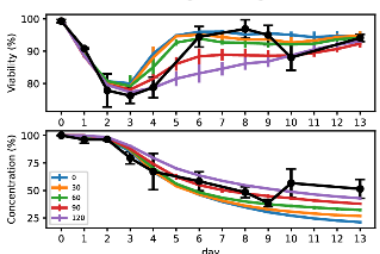

NLC Threshold

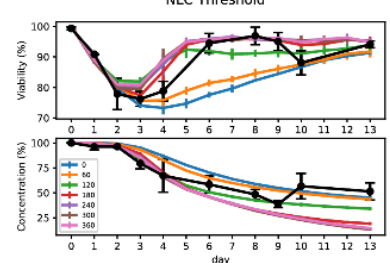

Differentiation Time
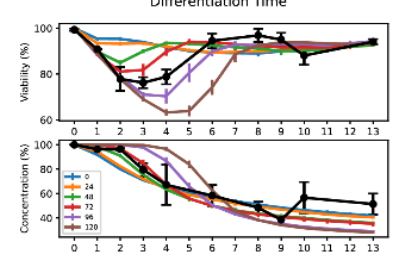
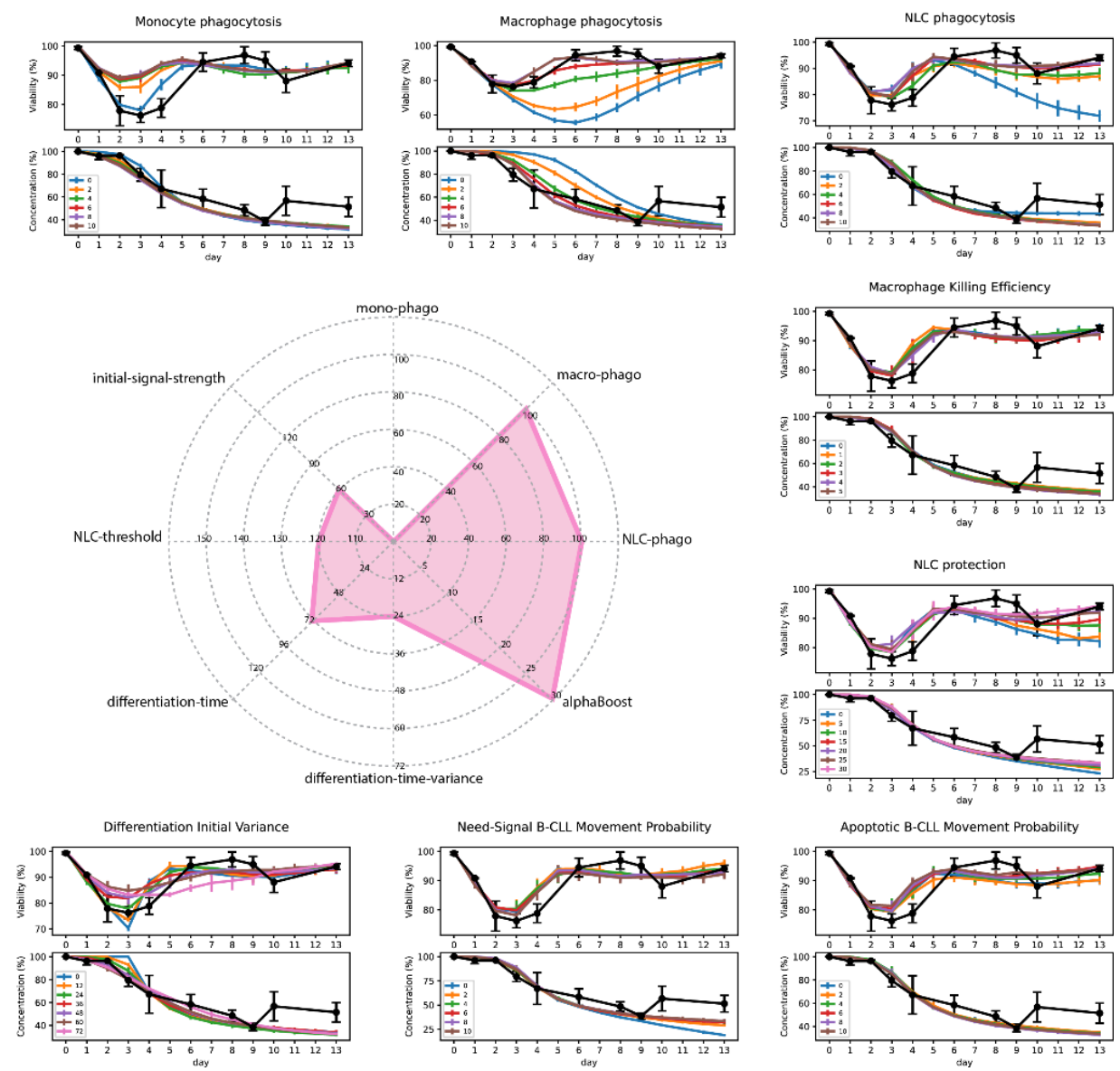
Fig. 4. Comparison of simulated and experimental time courses and parameter sensitivity analysis. A) 100 simulations were run with the best set of parameters and compared with the averaged viability and concentration observed experimentally. The initial monocytes proportion for the simulations was set to $1.6 \%$ corresponding to the average monocytes proportion measured in the patients. Simulations are depicted in red and experimental data in black. B) Simulations were run for varying initial monocytes proportions (3 repetitions) for 9 days and are here compared to experimental observations in heterologous cultures with the corresponding initial conditions at 9 days. Red bars correspond to the simulations and black bars correspond to the experimental data. C) 100 simulations were run with the best set of parameters and compared with the averaged viability and concentration observed experimentally setting the initial monocyte proportion to the specific percentage of monocytes measured in each patient (1.1\%, 2.5\% and 1.25\%). Simulations are depicted in red and experimental data for the corresponding patient in black. D) Parameter sensitivity analysis. Several parameters were varied one at a time while keeping all other parameters constant to estimate the impact of each parameter on the overall dynamics. The radial plot in the centre shows the optimal values of each parameter as selected by the parameter exploration.

\section{Discussion}

The presence of TAMs in solid tumours hinders the efficacy of immunotherapy, which in certain patients can be extremely beneficial. Given the difficulty of studying cellular dynamics in tissues, we turned to an in-vitro model of leukemia in which we can closely follow the production of TAMs from monocytes in presence with cancer cells.

Building on experimental measurements of cell numbers and viability of cancer cells in cultures of blood from Chronic Lymphocytic Leukemia patients, we implemented a two-dimensional agent-based model to simulate intercellular interactions in the spatial context of an in-vitro culture. More specifically, we modelled the interaction between cancer cells and monocytes, and the resulting differentiation of monocytes into macrophages that protect cancer cells (Nurse-Like Cells in this pathology). Using our experimental observations of viability and concentration of cells in a time course to perform parameter optimization and calibration, , we reproduced biologically realistic system dynamics and tested the model predictive power by simulating specific quantitative dynamics of NLC production varying the initial proportion of monocytes in co-cultures of monocytes from healthy donors with CLL cells. These observations suggest that models could be made patient-specific using this routinely measured variable and we show that using the initial monocyte concentration as input we can reproduce patient-specific dynamics for the 3 patients considered. Finally, we analyzed the impact of the different parameters and suggest a strong role for phagocytosis of monocytes and NLCs in the survival of cancer cells in this in-vitro CLL model, especially in the initial phases of the time course. The protective anti-apoptotic signals that have been known to be provided by NLCs to the cancer cells appear to be important especially towards the later stages of the culture. This finding suggests that monitoring and potentially modulation of phagocytosis could play a role in the control of tumour associated macrophage formation even in solid tumours. 
There are some important limitations of this study that we will list below. As far as the experiments are concerned, these in-vitro cultures might not fully represent what is happening in the patient lymph nodes, where the density of CLL cells is as high as what we reproduce in the cultures but potentially different cell populations other than cancer cells and monocyte/macrophages can be present in different proportions and the physical environment could be different. For example, in our cultures monocytes differentiate into macrophages in contact with the culture plate, something which is clearly artificial. Moreover, to be able to explore the impact of initial monocyte concentrations and thus validate the predictive power of our model, heterologous cultures of healthy monocyte donors and patient CLL cells were used. On one side, the interactions between cells from different subjects could introduce a cross-reaction of incompatibility, and on the other side we might be introducing effects due to the specific (epi)genetic characteristics of the donors.

Heterogeneity across patients is well known and the production of NLCs is extremely patient/time specific in both time scales and number of NLC produced. We are aware that different dynamics could be at play in different patients, and we hope that clinically measurable parameters, like the initial monocyte concentration in the blood and others, could help us produce a generally useful model that can give specific predictions. However, this involves making strong assumptions about the fact that the processes at play are common to different patients, which remains to be verified.

Regarding the ABM, we decided to model the system in two-dimensions, since the macrophages are adherent to the plate and most of the dynamics is determined by what happens on this surface. One of the main advantages of ABMs against other types of models is the ease with which spatial information can be integrated. We therefore plan to expand our experiment to be able to capture spatial patterns in the culture that can be easily compared to those appearing in our simulations. The initialization of these simulations is currently stochastic and does not take into account any information that could be extracted from high content imaging of the cultures. Despite this interesting prospect, the high density of cancer cells in the cultures have made quantitative imaging particularly difficult to achieve and we will explore these possibilities in further work.

An obvious extension of this model would involve simulating cellular dynamics in tissues, thus tackling the issue of TAM formation in solid tumours. We have recently developed tools to extract spatial information from tumour tissue samples either imaged via microscopy or characterised via single-cell spatial-omics techniques and we plan to establish a framework to use spatial data to initialize the ABM simulations in these more complex contexts.

Finally, we realize the importance of internal regulatory processes that determine the agents' behaviours and plan to extend this model by combining it with gene regulatory network models 
of phenotype transitions inside each cell (cancer cells, monocytes and potentially other cells presented in the TME of solid tumours in considerable proportions such as lymphocytes fibroblasts etc.). More specifically, we have been working on a Boolean model of monocyte differentiation into NLCs that we plan to integrate to the presented ABM model using suitable tools in further work.

\section{Materials and Methods}

Cell culture. Blood samples were obtained after informed consent and stored at the HIMIP collection. According to French law, the HIMIP collection was declared to the Ministry of Higher Education and Research (DC 2008-307 collection 1) and a transfer agreement (AC 2008-129) was agreed after approbation by the "Comité de Protection des Personnes Sud-Ouest et Outremer II" (ethical committee). Clinical and biological annotation of samples were declared to the Comité National Informatique et Libertés (CNIL; data processing and liberties national committee). PBMCs were isolated from the blood of CLL patients or buffy-coat from the healthy donors (HD). To generate autologous NLC, PBMC from CLL patient were cultured at $107 \mathrm{c} / \mathrm{mL}$ in RPMI 1640 supplemented with 10\% Fetal Calf Serum and and 1\% Penicillin/Streptomycin (Gibco) in the incubator at $37^{\circ} \mathrm{C}$ with $5 \% \mathrm{CO} 2$. Cells were cultured for 13 days during which differentiation of the NLC was followed by BFI microscopy and phenotype of the cells was assessed by flow cytometry (presence of CD14, CD163 and CD206) at the final day of the culture. At the indicated time points B-CLL cells samples were taken to measure cell concentration by hemocytometer and cell viability by flow cytometry using AnnexinV/7-AAD staining. To generate heterologous co-culture, B-CLL from PBMC fraction were isolated using negative selection (EasySep ${ }^{\mathrm{TM}}$ Human B Cell Enrichment Kit II Without CD43 Depletion, STEMcell) and monocytes from HD PBMC were isolated using positive selection method(CD14 MicroBeads, human, Miltneyi). Subsequently B-CLL cells at $107 \mathrm{c} / \mathrm{mL}$ were mixed with varying concentrations of purified monocytes. At day 9 concentration and viability of the B-CLL cells was measured and the phenotype of NLC was assessed by FACS. CLL PBMC used for the experiment contained $>85 \%$ of B-CLL cells and $0,5-2 \%$ of monocytes as assessed by flow cytometry. Purity of the isolated B-CLL and monocytes exceeded $95 \%$.

Agent Based Model. The ABM was implemented in NetLogo (24), mimicking a projection of a cylindrical plate. In general, macrophages and NLCs perform most of their functions after adherence, therefore the model was built in 2D. The simulation duration was set to 13 days with each simulation time step corresponding to 1 hour. At each time step, the following processes are executed by each cell in the following order: 
- update-position: movements are either random or directional (e.g. cancer cells move towards NLC, macrophages move towards dying cancer cells). Movements concern all cells except the dead ones.

- update-phagocytosis: this concerns the movement of a myeloid cell onto a cancer cell within a specific perception radius (or sensing-distance). If the myeloid cell encounters a dead or late-apoptotic cancer cell, it will phagocyte it with characteristic efficiencies (phagocytosis-efficiency), and the phagocytosed cancer cell is removed from the simulation. If the myeloid cell does not find a cell to phagocyte within its perception radius, it will move randomly to a surrounding patch with its characteristic moving probability. Additionally, macrophages $M 2 x$ are able to phagocyte living B-CLL cells in the need-signal state with a specific killing-efficiency.

- update-properties: increases or decreases life value for cancer cells $(+1,-1$, +alphaBoost), signal-strength for myeloid cells $(+1,-1)$, or the amount of anti-apoptotic signals on patches $(+1,-1$, diffusion).

- update-class: based on their properties values and specific calibrated thresholds, cells might change their state (class) at the next time step. For example, an apoptotic cancer cell will convert to dead if its life value goes below a certain threshold (death-threshold), need-signal cancer cells will turn into apoptotic cells if their life goes below apoptosis-threshold. Myeloid cells will differentiate from monocyte to M2X depending on their differentiation timing, and polarize from $M 2 x$ to $N L C$ or from $N L C$ to $M 2 x$, depending on their signal-strength compared to nlc-threshold.

For each cell state (class), the following actions are considered :

- Monocytes can perform 2 actions: differentiate into macrophages $M 2 x$ and perform phagocytosis. At the start of the simulation, monocytes start to adhere and differentiate into macrophages in a time window spanning from $60 \mathrm{~h}$ to $96 \mathrm{~h}$ (differentiationTime). Variability in this process is also explored (differentiationTimeDeviation) Monocytes can move towards apoptotic or dead B-CLL cells in their perception radius (monocyteSensingDistance), and will phagocyte these cells with their characteristic efficiency (mono-phago-eff).

- Macrophages can perform 3 actions: phagocytosis, polarization and killing the living (need-signal) B-CLL cancer cells. Similarly to monocytes, they can move towards apoptotic cells in their perception radius (macrophageSensingDistance) and phagocyte them with their characteristic efficiency (macro-phago-eff), whereas the killing of living B-CLL cells (need-signal) is performed with another characteristic efficiency 
(macro-kill-eff). At each time step, the number of contacts between macrophages and B-CLL cells (need-signal and late-apoptotic) is counted and the polarization into NLC occurs when the macrophages have encountered enough B-CLL cells (nlc-threshold). The polarization process is reversible.

- NLCs can perform 3 actions: phagocytosis of late-apoptotic or dead B-CLL cells, secretion of anti-apoptotic signals (referred to as chemical-A on patches) and re-polarization into $M 2 x$. Similar to $M 2 x$ macrophages, $N L C$ will move in the direction of apoptotic and dead CLL cells in their perception radius (nlcSensingDistance) and will phagocyte them with their characteristic efficiency (nlc-phago-eff). If the NLCs are not surrounded anymore by a sufficient amount of (need-signal) cancer cells, their signal-strength will decrease and they will eventually re-polarize into $M 2 x$ if it goes below nlc-threshold.

- B-CLL cancer cells can be in 3 possible states (need-signal, apoptotic or dead), each of which will influence the cancer cell's behaviour. In the need-signal state, B-CLL cells search for NLCs around them in a characteristic perception radius (CLLSensingDistance) and will move towards that direction, or randomly in the absence of surrounding NLCs. When B-CLL cancer cells find NLCs they will remain attached to them on $n$ layers (with $n$ being the number of layers of B-CLL that NLC can have around them - layersAroundNLC). Importantly, when B-CLL cancer cells move on patches containing anti-apoptotic signals (chemical-A), they consume a part of it, and get an increment of alpha-boost on their life property value, which helps them fight apoptosis and survive longer. In the late-apoptotic state, the cancer cells can no longer benefit from the anti-apoptotic signals from NLCs and will subsequently die. They can be phagocytosed. In the dead state, cells can no longer move. They also can be phagocytosed.

States and counters are updated at each time step. NLC-threshold is a proxy for the number of accumulated hours for which a Macrophage has been in contact with at least one need-signal cancer cell. We assumed that apoptotic and dead cells are phagocytosed with the same efficiency by macrophages. In the model, apoptotic cells move with a calibrated probability (from $01 \%$ to $1001 \%$ ) whereas dead cells do not move at all. Details about the parameter search can be found in Supplementary material. 


\section{References}

1. Alessandro Palma, Abdul Salam Jarrah, Paolo Tieri, Gianni Cesareni, and Filippo Castiglione. Gene regulatory network modeling of macrophage differentiation corroborates the continuum hypothesis of polarization states. Frontiers in physiology, 9:1659, 2018.

2. Fernando $\mathrm{O}$ Martinez and Siamon Gordon. The $\mathrm{m} 1$ and $\mathrm{m} 2$ paradigm of macrophage activa-tion: time for reassessment. F1000prime reports, 6, 2014.

3. Roy Noy and Jeffrey W Pollard. Tumor-associated macrophages: from mechanisms to ther-apy. Immunity, 41(1):49-61, 2014.

4. Alberto Mantovani, Paola Allavena, and Antonio Sica. Tumour-associated macrophages as a prototypic type ii polarised phagocyte population: role in tumour progression. European journal of cancer, 40(11):1660-1667, 2004.

5. F Boissard, JJ Fournie, A Quillet-Mary, L Ysebaert, and M Poupot. Nurse-like cells mediate ibrutinib resistance in chronic lymphocytic leukemia patients. Blood cancer journal, 5(10): e355-e355, 2015.

6. Elisa Ten Hacken and Jan A Burger. Microenvironment interactions and b-cell receptor sig-naling in chronic lymphocytic leukemia: Implications for disease pathogenesis and treatment. Biochimica et Biophysica Acta (BBA)-Molecular Cell Research, 1863(3):401-413, 2016.

7. Malvina Marku, Nina Verstraete, Flavien Raynal, Miguel Madrid-Mencía, Marcin Domagala, Jean-Jacques Fournié, Loïc Ysebaert, Mary Poupot, and Vera Pancaldi. Insights on tam for-mation from a boolean model of macrophage polarization based on in vitro studies. Cancers, 12(12):3664, 2020.

8. Li Jia, Andrew Clear, Feng-Ting Liu, Janet Matthews, Nadiha Uddin, Aine McCarthy, Elena Hoxha, Catherine Durance, Sameena Iqbal, and John G Gribben. Extracellular hmgb1 pro-motes differentiation of nurse-like cells in chronic lymphocytic leukemia. Blood, The Journal of the American Society of Hematology, 123(11):1709-1719, 2014.

9. $\quad$ F Boissard, C Laurent, AG Ramsay, A Quillet-Mary, JJ Fournie, M Poupot, and L Ysebaert. Nurse-like cells impact on disease progression in chronic lymphocytic leukemia. Blood cancer journal, 6(1):e381-e381, 2016.

10. Jan A Burger, Meike Burger, and Thomas J Kipps. Chronic lymphocytic leukemia b cells express functional cxcr4 chemokine receptors that mediate spontaneous migration beneath bone marrow stromal cells. Blood, The Journal of the American Society of Hematology, 94 (11):3658-3667, 1999.

11. Jan A Burger and Thomas $\mathrm{J}$ Kipps. Chemokine receptors and stromal cells in the homing and homeostasis of chronic lymphocytic leukemia b cells. Leukemia \& lymphoma, 43(3):461-466, 2002.

12. Hugo Talbot, Sofiane Saada, Elodie Barthout, Paul-François Gallet, Nathalie Gachard, Julie Abraham, Arnaud Jaccard, Danielle Troutaud, Fabrice Lalloué, Thomas Naves, et al. Bdnf be-longs to the nurse-like cell secretome and supports survival of b chronic lymphocytic leukemia cells. Scientific reports, 10(1):1-9, 2020.

13. Mitsufumi Nishio, Tomoyuki Endo, Nobuhiro Tsukada, Junko Ohata, Shinichi Kitada, John C Reed, Nathan J Zvaifler, and Thomas $\mathrm{J}$ Kipps. Nurselike cells express baff and april, which can promote survival of chronic lymphocytic leukemia cells via a paracrine pathway distinct from that of sdf-1

. Blood, 106(3):1012-1020, 2005.

14. Charles M Macal and Michael J North. Agent-based modeling and simulation. In Proceedings of the 2009 Winter Simulation Conference (WSC), pages 86-98. IEEE, 2009.

15. Angela Reynolds, Jonathan Rubin, Gilles Clermont, Judy Day, Yoram Vodovotz, and G Bard Ermentrout. A reduced mathematical model of the acute inflammatory response: I. derivation of model and analysis of anti-inflammation. Journal of theoretical biology, 242(1):220-236, 2006.

16. Alva Presbitero, Emiliano Mancini, Ruud Brands, Valeria V Krzhizhanovskaya, and Peter Sloot. Supplemented alkaline phosphatase supports the immune response in patients under-going cardiac surgery: clinical and computational evidence. Frontiers in immunology, 9:2342, 2018. 
bioRxiv preprint doi: https://doi.org/10.1101/2021.12.17.473137; this version posted December 20, 2021. The copyright holder for this preprint (which was not certified by peer review) is the author/funder, who has granted bioRxiv a license to display the preprint in perpetuity. It is made available under aCC-BY 4.0 International license.

17. Zarifeh Heidary, Jafar Ghaisari, Shiva Moein, and Shaghayegh Haghjooy Javanmard. The double-edged sword role of fibroblasts in the interaction with cancer cells; an agent-based modeling approach. PloS one, 15(5):e0232965, 2020.

18. Gary An, Qi Mi, Joyeeta Dutta-Moscato, and Yoram Vodovotz. Agent-based models in trans-lational systems biology. Wiley Interdisciplinary Reviews: Systems Biology and Medicine, 1 (2):159-171, 2009.

19. Zhihui Wang, Joseph D Butner, Romica Kerketta, Vittorio Cristini, and Thomas S Deisboeck. Simulating cancer growth with multiscale agent-based modeling. In Seminars in cancer biol-ogy, volume 30, pages 70-78. Elsevier, 2015.

20. Reinhard Laubenbacher, Franziska Hinkelmann, and Matt Oremland. Agent-based models and optimal control in biology: a discrete approach. Mathematical concepts and methods in modern biology, pages 143-178, 2013.

21. Erin N Bodine, Robert M Panoff, Eberhard O Voit, and Anton E Weisstein. Agent-based modeling and simulation in mathematics and biology education. Bulletin of Mathematical Biology, 82(8):1-19, 2020.

22. Ahmadreza Ghaffarizadeh, Randy Heiland, Samuel H Friedman, Shannon M Mumenthaler, and Paul Macklin. Physicell: An open source physics-based cell simulator for 3-d multicellular systems. PLoS computational biology, 14(2):e1005991, 2018.

23. Gaelle Letort, Arnau Montagud, Gautier Stoll, Randy Heiland, Emmanuel Barillot, Paul Mack-lin, Andrei Zinovyev, and Laurence Calzone. Physiboss: a multi-scale agent-based modelling framework integrating physical dimension and cell signalling. Bioinformatics, 35(7):1188-1196, 2019.

24. Elizabeth Sklar. Netlogo, a multi-agent simulation environment. Artificial life, 13(3):303-311, 2007.

25. Seth Tisue and Uri Wilensky. Netlogo: A simple environment for modeling complexity. In International conference on complex systems, volume 21, pages 16-21. Boston, MA, 2004.

26. Romain Reuillon, Mathieu Leclaire, and Sebastien Rey-Coyrehourcq. Openmole, a work-flow engine specifically tailored for the distributed exploration of simulation models. Future Generation Computer Systems, 29(8):1981-1990, 2013.

27. Kalyanmoy Deb. Evolutionary algorithms. In Multi-objective optimization using evolutionary algorithms, pages 81-170. John Wiley \& Sons, Ltd, 2001.

28. Kalyanmoy Deb, Amrit Pratap, Sameer Agarwal, and TAMT Meyarivan. A fast and elitist multiobjective genetic algorithm: Nsga-ii. IEEE transactions on evolutionary computation, 6 (2):182-197, 2002. 